\title{
ON A MONOTONE SINGULAR FUNCTION AND ON THE APPROXIMATION OF ANALYTIC FUNCTIONS BY NEARIY ANALYTIC FUNCTIONS IN THE COMPLEX DOMAIN
}

\author{
BY \\ H. KOBER
}

The classical example of a monotone continuous function $y=\omega(t)$ which is singular $\left({ }^{1}\right)$ was generalized by $\mathrm{T}$. Carleman $\left({ }^{2}\right)$. It was discussed in detail by E. Hille and J. D. Tamarkin $\left.{ }^{3}\right)$, it was generalized further by R. E. Gilman $\left({ }^{4}\right)$ who dealt with the intricate properties of $\omega^{\prime}(t)$; the method of these writers was based on binary, ternary, or higher scales of notation. In the present paper the Carleman-Gilman function will be generalised further still and dealt with from two new points of view:

I. The inverse function of $y=\omega(t)=\omega(t ; \alpha, \beta)$ admits of a plain representation

$$
\begin{aligned}
t=G(y)=q \sum_{\substack{m>-\infty \\
n \beta^{-m}<\sum^{\prime}<y}}^{\infty} \sum^{\infty} \alpha^{-m}=q \sum_{m>-\infty}^{\infty} a_{m, y} \alpha^{-m} \\
\quad(0 \leqq y<\infty ; m>-\log y / \log \beta)
\end{aligned}
$$

where $\beta$ is an integer, $\alpha>\beta \geqq 2, q=(\alpha-\beta)(\beta-1)^{-1}$, and where the dash indicates that $n / \beta$ should not be an integer; $a_{m, y}$ is the number of positive integers $n$, not divisible by $\beta$ and such that, given $m, n \beta^{-m}<y$. From (A) the LebesgueHille-Tamarkin example is obtained by taking $\alpha=3, \beta=2, y \leqq 1$, the Carleman functions by setting $\beta=2, \alpha=3$ or $4,5, \cdots ; y \leqq 1$, the Gilman example by taking $y \leqq 1, \alpha$ as an integer.

II. The function $\omega(t)=\omega(t ; \alpha, \beta)$ is completely defined by a few trivial conditions and by two functional equations one of which is known in the very special case $\beta=2, \alpha=3\left(^{5}\right)$. Again it satisfies three basic inequalities

$$
\begin{array}{rlrl}
\omega(t+\tau) & \leqq \omega(t)+\omega(\tau) & (t \geqq 0, \tau \geqq 0), \\
\text { (i) } \omega(t) \leqq t^{\lambda} ; & \text { (ii) } \omega(t) \geqq\left(\frac{\beta-1}{\alpha-1} t\right)^{\lambda}\left(\lambda=\frac{\log \beta}{\log \alpha} ; 0 \leqq t<\infty\right) .
\end{array}
$$

Presented to the Society, April 30, 1949; received by the editors August 30, 1948. 1928.

(1) That is, $\omega^{\prime}(t)=0$ for almost all $t$. H. Lebesgue, Lȩ̧ons sur l'intégration, 2d ed., Paris,

(2) Sur les équations intégrales singulières à noyau réel et symétrique, Uppsala Universitets Årsscrift, 1923, No. 3, pp. 223-226.

(3) Amer. Math. Monthly vol. 36 (1929) pp. 255-264.

(4) Ann. of Math. (2) vol. 33 (1932) pp. 433-442.

(5) G. H. Hardy and W. Rogosinski, Fourier series, Cambridge Tracts, no. 38, 1944, p. 27, line 8 from bottom. 
This settles finally the problem of the Lipschitz condition $\left({ }^{6}\right)$, as (B) and (C) imply that

$$
0 \leqq \omega(t+h)-\omega(t) \leqq h^{\lambda} \quad(0 \leqq t<\infty ; 0 \leqq h<\infty) .
$$

There is equality for an infinity of values of $t, \tau, h$. Thus (B)-(D) are best possible results. In connection with problem II the Fourier-Stieltjes transform of $\omega(t)(0 \leqq t \leqq 1)$ is computed $\left({ }^{7}\right)$.

In the second section of the present paper analytic functions are approximated by nearly analytic functions. The analytic functions are required to belong to the class $\Lambda$; this is the set of functions $f(z)$ which, for $|z|<1$, are analytic and such that $f^{\prime}(z)$ is bounded. The nearly analytic functions satisfy a Lipschitz condition in the region on which they are defined and are, therefore, continuous; their differential coefficient exists in the familiar sense at all points except for a set of surface measure zero. Obviously there are considerable differences between them and analytic functions, for instance with regard to their quasi-poles. They are constructed by means of the function $\omega(t)$. Their theory is given in some detail, since possibly they are of interest in themselves.

Given any two elements $g(z)$ and $h(z)$ of $\Lambda$, there exist nearly analytic functions $H_{n}(z)(n=1,2,3, \cdots)$ such that, uniformly for $|z| \leqq 1, H_{n}(z) \rightarrow g(z)$ as $n \rightarrow \infty$, while $H_{n}^{\prime}(z)=h^{\prime}(z)$ for all $n$ and almost all $z(\$ 2.7)$; explicit formulae are proved for the approximating functions.

Finally results are stated on the approximation of continuous functions of a real variable by "basic functions of bounded variation" $\left(^{8}\right)$.

1. The function $\omega(t)$. In this section the main properties of the functions $G(y)$ and $\omega(t)$ are proved.

1.1. The inverse function of $y=\omega(t)$ and the functional equations. The function $t=G(y)$, defined in $(0, \infty)$ by $(\mathrm{A})$, is a jump-function, with discontinuities at the rational points $y_{m, n}=n \beta^{-m}(m=0, \pm 1, \pm 2, \cdots$; $n=1,2, \cdots ; n / \beta$ not an integer), and with jump $q \alpha^{-m}$ at $y_{m, n}$. The points $\left\{y_{n, m}\right\}$ are dense everywhere in $(0, \infty)$. Hence $G(y)$ increases strictly, and

(6) Cf. Hille and Tamarkin, loc. cit. p. 259, and Gilman, loc. cit. Theorem 2. For $\beta=2$, $t \leqq 1$, (D) follows from a known result. F. Hausdorff (Math. Ann. vol. 79 (1919) pp. 157-179), dealing with exterior measures of "fractional dimensions," associates an exterior measure of sets to any function satisfying some conditions of concavity, and so on, for instance to $t^{p}(0<p$ $<1)$. Then he constructs a function $\phi(t)$ which is closely connected with his exterior measure and is actually identical with $\omega(t ; \alpha, 2)$ when we take $p=\log 2 / \log \alpha, \xi_{n}$ (see p. 169) equal to $\alpha^{-n}$, and shows (pp. 170-173) that $\omega\left(t_{2}\right)-\omega\left(t_{1}\right) \leqq\left(t_{2}-t_{1}\right)^{p}\left(0 \leqq t_{1}<t_{2} \leqq 1\right)$. My thanks are due to Professor A. Zygmund for drawing my attention to this classical paper. p. 263.

( $\left.{ }^{7}\right)$ For the cases $\beta=2, \alpha=3,4, \cdots$ see Carleman, loc. cit. Cf. Hille and Tamar kin, loc. cit.

(8) See $\$ 2.8$ of the present paper. A non-decreasing function of this kind is called "Scarto elementare” by G. Vitali, Rend. Circ. Mat. Palermo vol. 46 (1922) pp. 388-408; §17. 
the inverse function $y=\omega(t)$ is nondecreasing, continuous, and $\operatorname{singular}\left({ }^{9}\right)$; it is constant on the intervals of an open set of measure $a$ for $0 \leqq t \leqq a$. We have

$$
\begin{aligned}
& G\left(y_{m, n}-\right)=G\left(y_{m, n}\right)=t_{m, n} ; \quad G\left(y_{m, n}+\right)=t_{m, n}+q \alpha^{-m}, \\
& \omega(t)=y_{m, n} \text { for } t_{m, n} \leqq t \leqq t_{m, n}+q \alpha^{-m} .
\end{aligned}
$$

From (A) we deduce immediately that

$$
G\left(\frac{y}{\beta}\right)=\frac{1}{\alpha} G(y) \quad(0 \leqq y<\infty) .
$$

Again we have

$$
G(0)=0 ; G(1)=q\left\{\frac{\beta-1}{\alpha}+\frac{\beta(\beta-1)}{\alpha^{2}}+\frac{\beta^{2}(\beta-1)}{\alpha^{3}}+\cdots\right\}=1,
$$

since $n \beta^{-m}<1, m>0$ and since there are $\beta^{m-1}(\beta-1)$ integers $n$ which are not divisible by $\beta$ and such that $0<n<\beta^{m}$. Hence, by (1.12), $G(y) \rightarrow \infty$ as $y \rightarrow \infty$.

Now we take $0<y<1, y \neq y_{m, n}$; then we have $m>0$,

$$
G(y)+G(1-y)=q \sum_{n \beta^{-m}<y}^{m, n} \alpha^{-m}+q \sum_{y<\nu \beta-m}^{m, \nu} \alpha^{-m}
$$

where $\nu \beta^{-m}=1-n \beta^{-m}, \nu=\beta^{m}-n$ is an integer, while $\nu / \beta$ is not, and $\nu \beta^{-m}<1$. Hence

$$
G(y)+G(1-y)=q \sum_{n \beta^{-m}<1}^{m, n} \alpha^{-m}=G(1)=1 \quad\left(y \neq y_{m, n} ; 0<y<1\right) .
$$

Finally, for $0<y<1-\beta^{-1}$, we have

$$
G\left(y+\frac{1}{\beta}\right)-G(y)=q \sum_{y \leqq n \beta-m<y+\beta^{-1}}^{\prime} \alpha^{-m} .
$$

Clearly $m>0$ as $y+\beta^{-1} \leqq 1$. The number of the $n$ 's, not divisible by $\beta$ and such that $\beta^{m} y \leqq n<\beta_{v}^{m}+\beta^{m-1}$, is unity for $m=1$, is $\beta^{m-2}(\beta-1)$ for $m \geqq 2$. Hence

$$
\begin{aligned}
G\left(y+\frac{1}{\beta}\right)-G(y)= & q\left\{\frac{1}{\alpha}+\sum_{m=2}^{\infty} \frac{\beta^{m-2}(\beta-1)}{\alpha^{m}}\right\} \\
& =\frac{\alpha-1}{\alpha(\beta-1)}=\frac{q+1}{\alpha} \quad\left(0<y<1-\frac{1}{\beta}\right) .
\end{aligned}
$$

From (1.12)-(1.15) we deduce the following properties of the inverse function $y=\omega(t)=\omega(t ; \alpha, \beta)$ :

( $\left.{ }^{9}\right) \mathrm{H}$. Kober, On singular functions of bounded variation, J. London Math. Soc. vol. 23 (1948) pp. 222-229. 

(a) $\omega(0)=0$,
(b) $\omega(1)=1$,
(c) $\omega(t / \alpha)=\omega(t) / \beta$
$(0 \leqq t<\infty)$
(d) $\omega\left(t+\frac{q+1}{\alpha}\right)=\omega(t)+\frac{1}{\beta}$
$\left(0 \leqq t \leqq 1-\frac{q+1}{\alpha}=1-\frac{\alpha-1}{\alpha(\beta-1)}\right)$,
(e) $\omega(t)+\omega(1-t)=1$
$(0 \leqq t \leqq 1)$,

observing that $\omega(t)$ is continuous and that $y+1 / \beta<1$ implies that $t+(q+1) / \alpha$ $<1$ and vice versa. From (d), or from (c) and (e), respectively, we deduce that

$$
\begin{aligned}
\text { (d') } \omega\left(t+j \frac{q+1}{\alpha}\right) & =\omega(t)+\frac{j}{\beta} \\
\left(j=1,2, \cdots \beta-1 ; 0 \leqq t \leqq 1-j \frac{q+1}{\alpha}\right), & \left(j=0, \pm 1, \pm 2, \cdots ; 0 \leqq t \leqq \alpha^{j}\right) .
\end{aligned}
$$

We notice that, for $\beta=2$, the equations (c) and (e) together imply (d), as

$$
\omega\left(t+1-\frac{1}{\alpha}\right)=1-\omega\left(\frac{1}{\alpha}-t\right)=1-\left(\frac{1}{2}-\omega(t)\right)=\omega(t)+\frac{1}{2}
$$

by $\left(\mathrm{e}^{\prime}\right)$, with $j=0$ and $j=-1$.

The equation (c) is known for $\alpha=3, \beta=2,0 \leqq t \leqq 1$, (e) is known for the case when $\alpha$ is an integer $\left({ }^{10}\right)$.

1.2. The Fourier-Stieltjes transform of $y=\omega(t)(0 \leqq t \leqq 1)$ and the functional equations. To show that, in substance, the function $y=\omega(t)$ is completely defined by functional equations, we prove the following theorem.

Theorem 1. If a function $y=\psi(t ; \alpha, \beta)(\alpha>\beta \geqq 2 ; \beta$ an integer) (i) does not decrease in some interval $0 \leqq t \leqq \delta$ and (ii) satisfies (1.16), (a)-(d), then it is identical with the function $y=\omega(t ; \alpha, \beta)$.

The proof is based on following theorem.

THEOREM 1'. Under the conditions of Theorem 1, the Fourier-Stieltjes transform

$$
F(x)=\int_{0}^{1} e^{i x t} d \psi(t)
$$

is

${ }^{10}$ Gilman, loc. cit. 


$$
F(x)=e^{i x / 2} \prod_{n=0}^{\infty} \frac{\sin \left(\beta x \alpha^{-n} \Delta / 2\right)}{\beta \sin \left(x \alpha^{-n} \Delta / 2\right)} \quad\left(\Delta=\frac{1-\alpha^{-1}}{\beta-1}\right) .
$$

REMARK. When $\beta=2$ then $F(x)$ takes the form

$$
F(x)=e^{i x / 2} \prod_{n=1}^{\infty} \cos \frac{(\alpha-1) x}{2 \alpha^{n}} .
$$

This was proved by Carleman for the case when $\alpha$ is an integer( $\left.{ }^{11}\right)$.

Proof. In virtue of (1.16c), (i) implies that $\psi(t)$ does not decrease in $(0, \infty)$. We now consider the $\beta-1$ closed intervals

$$
\begin{aligned}
& I_{j}=\langle 1 / \alpha+(j-1) \Delta, j \Delta\rangle=\left\langle a_{j}, b_{j}\right\rangle \\
& \quad\left(\Delta=\frac{\alpha-1}{\alpha(\beta-1)}=\frac{q+1}{\alpha} ; j=1,2, \cdots, \beta-1\right) .
\end{aligned}
$$

On each of these intervals $\psi(t)$ is constant. For $\psi\left(\alpha^{-1}\right)=\beta^{-1}$ by (b) and (c) while $\psi(\Delta)=\beta^{-1}$ by (a) and (d). Hence $\psi(t)=\beta^{-1}$ on $I_{1}$, and $\psi(t)=2 \beta^{-1}$ on $I_{2}$, $\psi(t)=j \beta^{-1}$ on $I_{j}$ in consequence of $\left(\mathrm{d}^{\prime}\right)$. Hence

$$
\int_{a_{j}}^{b_{j}} e^{i x(t-1 / 2)} d \psi(t)=0 \quad(j=1,2, \cdots, \beta-1) .
$$

Now we have

$$
\begin{array}{r}
U_{j}=\int_{b_{j}}^{a_{j}+1} e^{i x(t-1 / 2)} d \psi(t)=\int_{0}^{1 / \alpha} e^{i x(t+j \Delta-1 / 2)} d \psi(t) \\
\quad(j=0,1,2, \cdots, \beta-1),
\end{array}
$$

using $\left(\mathrm{d}^{\prime}\right)$ and taking $b_{0}=0, a_{\beta}=1$. Writing

$$
f(x)=\int_{0}^{1} e^{i x(t-1 / 2)} d \psi(t)
$$

and using (c), we deduce that

$$
U_{j}=\frac{1}{\beta} \int_{0}^{1} e^{i x(t / \alpha+j \Delta-1 / 2)} d \psi(t)=\frac{1}{\beta} \exp \left\{i x\left(j \Delta-\frac{\alpha-1}{2 \alpha}\right)\right\} f\left(\frac{x}{\alpha}\right) .
$$

Hence

$$
\begin{aligned}
& f(x)=\sum_{j=0}^{\beta-1} U_{j}=\beta^{-1} f\left(\frac{x}{\alpha}\right) \exp \left\{\frac{-i x(\alpha-1)}{2 \alpha}\right\} \sum_{j=0}^{\beta-1} \exp (i x j \Delta), \\
& f(x)=u(x) f\left(\frac{x}{\alpha}\right) ; \quad u(x)=\frac{\sin (x \beta \Delta / 2)}{\beta \sin (x \Delta / 2)}
\end{aligned}
$$

\footnotetext{
${ }^{11}$ Cf. footnote 7 .
} 
Thus

$$
\begin{aligned}
& f\left(\frac{x}{\alpha}\right)=u\left(\frac{x}{\alpha}\right) f\left(\frac{x}{\alpha^{2}}\right) ; \quad f\left(\frac{x}{\alpha^{n}}\right)=u\left(\frac{x}{\alpha^{n}}\right) f\left(\frac{x}{\alpha^{n+1}}\right)(n=0,1,2, \cdots), \\
& f(x)=\prod_{n=1}^{\infty} u\left(\frac{x}{\alpha^{n}}\right)=\prod_{n=0}^{\infty} \frac{\sin \left(\Delta \beta \alpha^{-n} x / 2\right)}{\beta \sin \left(\Delta \alpha^{-n} x / 2\right)} \quad\left(\Delta=\frac{\alpha-1}{\alpha(\beta-1)}\right),
\end{aligned}
$$

as $\alpha>2$ and, by (1.26), $f\left(x \alpha^{-n}\right) \rightarrow \psi(1)-\psi(0)=1(n \rightarrow \infty)$ for any $x$. This proves the theorem.

REMARK. It follows from (1.26) that $f(z)$ is an entire function and that, for $z=x+i y,|f(z)| \leqq \exp (|y| / 2)$.

Now the conditions of the theorem are satisfied by the function $y=\omega(t)$. Hence (1.22) holds for the Fourier-Stieltjes transform of this function. By a known uniqueness theorem and by the continuity of $y=\omega(t)$, the functions $\omega(t)$ and $\psi(t)$ are identical in $\langle 0,1\rangle$ and, by (1.16c), in $\langle 0, \infty\rangle$, which completes the proof of the main theorem.

1.3. The intervals of invariance of $\omega(t)$. Before dealing with the inequalities (B) and (C) we must investigate into the position of the intervals on which $y=\omega(t)$ is constant. Let $0 \leqq t \leqq 1$, let $y_{m, n}=n \beta^{-m}\left(m \geqq 1, n<\beta^{m}, n / \beta\right.$ not an integer), $t_{m, n}=G\left(y_{m, n}\right), t_{m, n}^{\prime}=t_{m, n}+q \alpha^{-m}=G\left(y_{m, n}+\right.$ ) (see 1.11). Then $\omega(t)$ is constant on the interval $I_{m, n}=\left\langle t_{m, n}, t_{m, n}^{\prime}\right\rangle$ which is said to be of order $m(m=1,2, \cdots)$. The intervals of order one are stated in (1.23). Denoting them now by $I_{1, j}(j=1,2, \cdots, \beta-1)$, we have

$$
y=y_{1, j}=\omega\left\{\frac{j(1+q)}{\alpha}\right\}=\frac{j}{\beta} \text { on } I_{1, j} \quad(j=1,2, \cdots, \beta-1) .
$$

The length of each of the $I_{m, j}$ is $q \alpha^{-m}$, their number is $\beta^{m}-\beta^{m-1}$ ( $m$ fixed). Thus

$$
\sum_{m=1}^{\infty} \sum_{j=1}^{\infty}\left|I_{m, j}\right|=\sum_{m=1}^{\infty} q \alpha^{-m}\left(\beta^{m}-\beta^{m-1}\right)=1
$$

All the intervals of order $k \leqq M-1(M=2,3,4, \cdots)$ can be found this way: set $\eta_{N}=N / \beta^{M-1}$ where $N$ runs through all the numbers $1,2, \cdots, \beta^{M-1}-1$. The number of these intervals is $\beta^{M-1}-1$; their end points are denoted by

$$
T_{M-1, N}=G\left(\eta_{N}\right), \quad T_{M-1, N}^{\prime}=G\left(\eta_{N}+\right) .
$$

Between any two neighbouring intervals of order $k \leqq M-1$, also between 0 and $T_{M-1,1}$, and between $T_{M-1, N-1}^{\prime}$ and $T_{M-1, N}=1$ for $N=\beta^{M-1}$, there are $\beta-1$ intervals of order $M$, with end points 


$$
\begin{aligned}
& \tau_{j}=G\left(\eta_{N}-j \beta^{-M}\right), \quad \tau_{j}^{\prime}=G\left(\eta_{N}-j \beta^{-M}+\right)=\tau_{j}+q \alpha^{-M} \\
&\left(M, N \text { fixed; } N=1,2, \cdots, \beta^{M-1} ; j=1,2, \cdots, \beta-1\right) .
\end{aligned}
$$

For $\eta_{N}-j \beta^{-M}=(N \beta-j) \beta^{-M}$, and $N \beta-j$ is not divisible by $\beta$. The length of each of these intervals is $q \alpha^{-M}$ by (1.32); writing $\eta$ for $\eta_{N}$ and using (A) we deduce that

$$
T_{M-1, N}-\tau_{1}^{\prime}=q \sum_{\mu, \nu ; \eta-\beta^{-M}<\nu \beta^{-\mu}<\eta}^{\alpha^{-\mu}}=q\left\{\frac{\beta-1}{\alpha^{M+1}}+\frac{\beta(\beta-1)}{\alpha^{M+2}}+\cdots\right\}=\frac{1}{\alpha^{M}} .
$$

For $\eta \beta^{M-1}$ is an integer, while there are no integers between $\eta \beta^{\mu}-\beta^{\mu-M}$ and $\eta \beta^{\mu}$ for $\mu \leqq M$, but $\beta^{l-1}(\beta-1)$ integers for $\mu=M+l(l=1,2, \cdots)$. Similarly

$\tau_{j}-\tau_{j+1}^{\prime}=q \sum_{\mu, \nu ; \eta-(j+1) \beta^{-M}<\nu \beta^{-\mu}<\eta-j \beta-M}^{\prime} \quad \alpha^{-\mu}=\frac{1}{\alpha^{M}} \quad(j=1,2, \cdots, \beta-1)$,

setting $\tau_{j+1}^{\prime}=T_{M-1, N-1}^{\prime}$ for $j=\beta-1, N>1 ; \tau_{j+1}^{\prime}=0$ for $j=\beta-1$ and $N=1$ (that is, $\eta_{N}=\eta_{1}=\beta^{-M+1}$ ). Thus we arrive at the following lemma.

LEMMA 1. In $\langle 0,1\rangle$ there are $\beta-1$ intervals of order $m(m>1)$, each of length $q \alpha^{-m}$, which lie between any two intervals of order smaller than $m$ or between the points $t=0$ and $t=\alpha^{-m+1}$ or between $t=1-\alpha^{-m+1}$ and $t=1$. The distance between any two neighbouring intervals of order not greater than $m(m \geqq 1)$ is $\alpha^{-m}$, the left or right end point of the outermost interval on the left or right is $t=\alpha^{-m}$ or $t=1-\alpha^{-m}$, respectively.

1.4. The inequality (B). We shall now show that for $0 \leqq t<\infty, 0 \leqq \tau<\infty$, the inequality (B) holds.

First we deal with a special case and prove that

$$
\omega\left(t+\frac{1+q}{\alpha}\right) \leqq \omega(t)+\omega\left(\frac{1+q}{\alpha}\right) \quad(0 \leqq t<\infty) .
$$

There is equality for $0 \leqq t \leqq 1-(1+q) \alpha^{-1}$. This follows from $(1.16 \mathrm{~d})$ as $\omega\left\{(1+q) \alpha^{-1}\right\}=\beta^{-1}$.

For $0<y<\infty$, we have

$$
G\left(y+\frac{1}{\beta}\right)-G(y)=q \sum_{y \leqq n \beta^{-m}<y+\beta^{-1}}^{\prime} \alpha^{-m} \geqq \sum_{y \leqq n \beta^{-m}<y+\beta^{-1} ; m>0}^{\prime} \alpha^{-m} .
$$

By the argument of the proof of (1.15), the latter series converges to $(1+q) \alpha^{-1}$. Hence

$$
G\left(y+\frac{1}{\beta}\right)-G(y) \geqq \frac{1+q}{\alpha}=G\left(\frac{1}{\beta}+\right) \quad(0<y<\infty) .
$$

As $\omega=G^{-1}$ is nondecreasing (1.42) implies (1.41). There is equality in (1.42) 
if, and only if, there is no positive integer $k$ such that $y \leqq k<y+\beta^{-1}$, that is, that $\omega(t) \leqq k<\omega(t)+\beta^{-1}$.

By (1.16c), (1.41) implies that

$$
\omega\left\{t+(1+q) \alpha^{-m}\right\} \leqq \omega(t)+\omega\left\{(1+q) \alpha^{-m}\right\} \quad(0 \leqq t<\infty) .
$$

Now we show that

$$
\omega\left(t+\zeta^{\prime}\right) \leqq \omega(t)+\omega\left(\zeta^{\prime}\right) \quad\left(0 \leqq t<\infty ; 0<\zeta^{\prime}<1\right)
$$

if $\zeta^{\prime}$ is the right end point of an interval $I_{m, j}$ (see 1.3). By (1.41), we have

$$
\begin{aligned}
\omega\left\{t+j \frac{(1+q)}{\alpha}\right\} \leqq \omega\left\{t+\frac{(j-1)(1+q)}{\alpha}\right\}+\omega\left(\frac{1+q}{\alpha}\right) & \\
& (j=1,2, \cdots, \beta-1),
\end{aligned}
$$

and repeating this procedure (cf. $\left.1.17 \mathrm{~d}^{\prime}\right)$ and using (1.31), we deduce (1.43) for the right end points $\zeta^{\prime}$ of the intervals of order one. Suppose now that it holds for the intervals of order smaller than $m(m \geqq 2)$. Let $\left\langle\xi, \xi^{\prime}\right\rangle\left\langle\xi^{\prime}<1\right)$ be the interval of order $m$ which is nearest the right end point of such an interval $\left\langle\zeta, \zeta^{\prime}\right\rangle$. Then we have, using Lemma 1 ,

$$
\xi=\zeta^{\prime}+\alpha^{-m} ; \quad \xi^{\prime}=\xi+q \alpha^{-m}=\zeta^{\prime}+(1+q) \alpha^{-m},
$$

and

$$
\omega\left(t+\xi^{\prime}\right) \leqq \omega\left(t+\zeta^{\prime}\right)+\omega\left\{(1+q) \alpha^{-m}\right\} \leqq \omega(t)+\omega\left(\zeta^{\prime}\right)+\beta^{-m},
$$

using (1.43) twice. But $\omega\left(\zeta^{\prime}\right)$ is of the form $N \beta^{1-m}=(N \beta) \beta^{-m}, \omega\left(\xi^{\prime}\right)$ of the form $N^{\prime} \beta^{-m}$. Hence, applying results of $\$ 1.3$ to the intervals of order not greater than $m-1$, we have $N^{\prime}=N \beta+1$

$$
\omega\left(\xi^{\prime}\right)=(N \beta+1) \beta^{-m}=\omega\left(\zeta^{\prime}\right)+\beta^{-m} .
$$

Combining (1.44) and (1.45) we arrive at (1.43). In a similar way we deal with the right end points of the other $\beta-2$ intervals of order $m$ which lie between $\left\langle\xi, \xi^{\prime}\right\rangle$ and the nearest interval of order not greater than $m-1$ on the right. Between $\tau=0$ and $\tau=\alpha^{-m+1}$ there are $\beta-1$ intervals of order $m$,

$$
I_{m, j}=\left\langle(j(1+q)-q) / \alpha^{m}, j(1+q) / \alpha^{m}\right\rangle \quad(j=1,2, \cdots, \beta-1),
$$

and (1.43) holds for their right end points $j(1+q) \alpha^{-m}$ by $\left(1.41^{\prime}\right)$. Hence (B) is true for the right end point $\tau$ of any interval $I_{m, j}$ in $(0,1)$. Since $\omega(t+\tau)$ is a nondecreasing function of $\tau$, (B) holds for all the points $\tau$ of such an interval and, by continuity, for any $\tau(0 \leqq \tau \leqq 1)$. Using (1.16c), we complete the proof.

REMARK. By the lemma $\left({ }^{12}\right): A$ function $y=f(t)$ of bounded variation $V_{0, a} f$

(12) H. Kober, loc. cit. Cf. T. Rado, Length and area, Amer. Math. Soc. Colloquium Publications, vol. 30, III.3.30; there $f(t)$ is supposed to be continuous. 
over $\langle 0, a\rangle$ is singular if and only if $L_{0, \alpha} f$, the length of the arc joining the points $\{0 ; f(0)\}$ and $\{a ; f(a)\}$, is equal to $a+V_{0, a} f$, the inequality $(\mathrm{B})$ is equivalent to $L_{0, t+\tau} \omega \leqq L_{0, t} \omega+L_{0, \tau} \omega$.

1.5. Two inequalities. To prove that $\omega(t) \leqq t^{\lambda}$ we need two lemmas:

Lemma 2. When $1<\beta<\alpha, 0 \leqq s \leqq 1$, then

$$
\{1+(\alpha-1) s\}^{\log \beta} \geqq\{1+(\beta-1) s\}^{\log \alpha} .
$$

LEMMA 3. When $1 \leqq j \leqq \beta, 0 \leqq s \leqq 1,0<\log \beta / \log \alpha=\lambda \leqq 1$, then

$$
(\alpha-j s)^{\lambda}-j(1-s)^{\lambda}-(\beta-j) \geqq 0 .
$$

To prove Lemma 2 we show that the function

$$
\phi(s)=\log \beta \log \{1+(\alpha-1) s\}-\log \alpha \log \{1+(\beta-1) s\}
$$

is not negative for $0 \leqq s \leqq 1$. We have $\phi(0)=\phi(1)=0$, and it is easily seen that $\phi^{\prime}(s)$ has not more than one zero. But $\phi^{\prime}(0)>0$ since

$$
\begin{aligned}
\psi(\alpha, \beta) & =(\alpha-1) \log \beta-(\beta-1) \log \alpha=\phi^{\prime}(0) ; \quad \psi(\beta, \beta)=0 . \\
\alpha \frac{\partial \psi(\alpha, \beta)}{\partial \alpha} & =\alpha \log \beta-\beta+1>\beta \log \beta-\beta+1=\int_{1}^{\beta} \log u d u>0 .
\end{aligned}
$$

Hence, $\phi(s)$ reaches a positive maximum in $(0,1)$ which proves the lemma.

To prove Lemma 3 we denote the function on the left by $g(s)$. We have

$$
(j \lambda)^{-1} g^{\prime}(s)=(1-s)^{\lambda-1}-(\alpha-j s)^{\lambda-1}
$$

and $g(0)=\alpha^{\lambda}-\beta=0$. The term on the right in (1.51) is not negative; for $\lambda-1 \leqq 0$, and $\alpha-j s \geqq 1-s$ as $\alpha-1 \geqq s(\beta-1) \geqq s(j-1)$. Thus we have proved the lemma.

1.6. The inequalities (C). To show that $\omega(t ; \alpha, \beta) \leqq t^{\lambda}(\lambda=\log \beta / \log \alpha$; $0 \leqq t<\infty)$ we need only prove this for the left end points of the intervals $I_{m, j}$ in $(0,1)$ (see 1.3). When the intervals are of order one we have $\omega\{(j+j q-q) / \alpha\}=j / \beta \quad(j=1,2, \cdots, \beta-1)$. Thus we must show that $i\left(\beta \leqq\{(j+j q-q) / \alpha\}^{\lambda}\right.$ or, as $\beta=\alpha^{\lambda}$, that

$$
j \leqq\left\{\frac{j(\alpha-1)-\alpha+\beta}{\beta-1}\right\}^{\lambda} .
$$

By the substitution $j=1+(\beta-1) s\left[0 \leqq s \leqq(\beta-2)(\beta-1)^{-1}\right],(1.61)$ reduces to the statement of Lemma 2.

Suppose now that in $(0,1)$ the inequality is true for the left end points of all the intervals of order smaller than $M$. Consider the $\beta-1$ intervals of order $M$ lying between neighbouring intervals

$$
\left\langle T_{0}, T_{0}^{\prime}\right\rangle,\left\langle T, T^{\prime}\right\rangle \quad\left(T_{0} \geqq \alpha^{1-M}>0 ; T_{0}^{\prime}=T-\alpha^{-M+1} ; T^{\prime}<1\right)
$$


of order smaller than $M$. By Lemma 1 , they are

(1.63) $\left\langle T-j(1+q) / \alpha^{M}, T-(j+(j-1) q) / \alpha^{M}\right\rangle(j=1,2, \cdots, \beta-1)$. If $\omega(T)=N \beta^{1-M}=(N \beta) \beta^{-M}$, then we have

$$
\begin{aligned}
& \omega\left(T-j \frac{1+q}{\alpha^{M}}\right)=\frac{N \beta-j}{\beta^{M}} ; \quad \omega\left(T_{0}\right)=\omega\left(T_{0}^{\prime}\right)=\frac{N-1}{\beta^{M-1}} \\
&(j=1,2, \cdots, \beta-1) .
\end{aligned}
$$

We shall show that

$$
\{N \beta-j) \beta^{-M} \leqq\left\{T-j(1+q) \alpha^{-M}\right\}^{\lambda} .
$$

Since $\omega(t) \leqq t^{\lambda}$ on the intervals of order smaller than $M$, we have

$$
N \beta^{1-M}=\omega(T) \leqq T^{\lambda} ;
$$$$
\text { (ii) }(N-1) \beta^{1-M}=\omega\left(T_{0}\right) \leqq\left\{T-(1+q) \alpha^{1-M}\right\}^{\lambda} \text {, }
$$

observing that $T_{0} \leqq T_{0}^{\prime}-q \alpha^{1-M}=T-(1+q) \alpha^{-M}$. Multiplying (1.65) (i) and (ii) by $\beta-j$ and $j$, respectively, and adding, we have

$$
\frac{N \beta-j}{\beta^{M-1}} \leqq(\beta-j) T^{\lambda}+j\left(T-\frac{1+q}{\alpha^{M-1}}\right)^{\lambda} \quad(j=1,2, \cdots, \beta-1) .
$$

Hence we need show that

$$
\beta\left\{T-j(1+q) \alpha^{-M}\right\}^{\lambda}-(\beta-j) T^{\lambda}-j\left\{T-(1+q) \alpha^{1-M}\right\} \geqq 0 .
$$

But this inequality follows from Lemma 3 taking $s=(1+q) \alpha^{1-M} T^{-1}$. The proof holds when $T$ is replaced by unity. Again for $T=\alpha^{1-M}$ there is no interval of order smaller than $M$ on the left; the intervals of order $M$ between $t=0$ and $T$ are stated above (see 1.46), and the result for their left end points follows from that on the intervals of order one by means of (1.16c). Hence $\omega(t) \leqq t^{\lambda}$ on all the intervals of order $M$ for any $M(M=1,2, \cdots)$. By continuity, the inequality holds for $0 \leqq t \leqq 1$, and by (1.16c) for $0 \leqq t<\infty$; which completes the proof of $(\mathrm{Ci})$; that of (Cii) is similar.

REMARK. From the inequalities (B)-(D) we can deduce results on the jump-function $G(y)$, defined by (A); observing that $G(y)=G(y-)$ :

$$
\begin{array}{lr}
G(y+z) \geqq G(y)+G(z) & (0 \leqq y<\infty, 0 \leqq z<\infty) . \\
\frac{\alpha-1}{\beta-1} y^{1 / \lambda} \geqq G(y) \geqq y^{1 / \lambda} & \left(\lambda=\frac{\log \beta}{\log \alpha} ; 0 \leqq y<\infty\right) . \\
G(y+h)-G(y) \geqq h^{1 / \lambda} & (0 \leqq y<\infty, 0 \leqq h<\infty ; \lambda=\log \beta / \log \alpha) .
\end{array}
$$

2. The nearly analytic functions. In this section the approximation of elements of $\Lambda$ by nearly analytic functions will be treated. We start with

2.1. An inequality. 
LEMma 4.

$$
|\omega(t ; \alpha, \beta)-t| \leqq 2 / \beta-2 / \alpha \quad \text { for } 0 \leqq t \leqq 1 .
$$

This need be proved only for the right end points of the intervals on which $\omega(t)$ is constant, or for the left end points. For if $\left\langle T, T^{\prime}\right\rangle$ is such an interval, then, by $(1.16 \mathrm{e}),\left\langle 1-T^{\prime}, 1-T\right\rangle$ is an interval of the same kind. And if the inequality is true for the left end points $T$ and $1-T^{\prime}$, then it holds for the right end points as, by $(1.16 \mathrm{e})$

$$
\omega\left(T^{\prime}\right)-T^{\prime}=1-\omega\left(1-T^{\prime}\right)-T^{\prime}=\left(1-T^{\prime}\right)-\omega\left(1-T^{\prime}\right) .
$$

Since $\omega(t)$ is constant on $\left\langle T, T^{\prime}\right\rangle$, the inequality holds for $T \leqq t \leqq T^{\prime}$. As the sum of the lengths of all these intervals is unity and as $\omega(t)$ is continuous, the inequality is true for $0 \leqq t \leqq 1$.

The right end points of the intervals of order one are (see $1.23 ; T_{j}^{\prime}=b_{j}$ )

$$
T_{i}^{\prime}=G\left(\frac{j}{\beta}+\right)=G\left(\frac{j}{\beta}\right)+\frac{1}{\alpha}=\frac{j(\alpha-1)}{\alpha(\beta-1)} \quad(j=1,2, \cdots, \beta-1) .
$$

Hence

$$
\left|\omega\left(T_{j}^{\prime}\right)-T_{j}^{\prime}\right|=\left|\frac{j}{\beta}-\frac{j(\alpha-1)}{\alpha(\beta-1)}\right|=\frac{j}{\beta-1}\left(\frac{1}{\beta}-\frac{1}{\alpha}\right) \leqq \frac{1}{\beta}-\frac{1}{\alpha} .
$$

Let $T$ or $T^{\prime}$, respectively, be the left or right end point of an interval of order not exceeding $m-1(m>1), \omega(T)=N / \beta^{m-1}$ (cf. $\left.\S 1.3 ; \omega(t)=\eta_{N}\right)$ where $N=1$, or $=2,3, \cdots, \beta^{m-1}-1$, or let $T=1$, with $N=\beta^{m}$, and suppose that, uniformly with respect to $N$,

$$
|\omega(T)-T| \leqq M_{m-1}
$$

On the left of $T$, between $T$ and the nearest interval of order not greater than $m-1$, or between $T$ and $t=0$ when $N=1$, there are $\beta-1$ intervals of order $m$, with left end points

$$
t_{k}=G\left(N \beta^{1-m}-k \beta^{-m}\right)=T-k \frac{\alpha-1}{\alpha^{m}(\beta-1)} \quad(k=1,2, \cdots, \beta-1) .
$$

Hence

$$
\begin{aligned}
\left|\omega\left(t_{k}\right)-t_{k}\right| & =\left|\frac{N \beta-k}{\beta^{m}}-\left\{T-k \frac{\alpha-1}{\alpha^{m}(\beta-1)}\right\}\right| \\
& \leqq\left|T-\frac{N}{\beta^{m-1}}\right|+k\left|\frac{1}{\beta^{m}}-\frac{\alpha-1}{\alpha^{m}(\beta-1)}\right| \\
& \leqq M_{m-1}+\frac{\beta-1}{\beta^{m}}-\frac{\alpha-1}{\alpha^{m}}
\end{aligned}
$$


if we use (2.12) and observe that $(\beta-1) \beta^{-m}-(\alpha-1) \alpha^{-m}>0$ for $m \geqq 2, \alpha>\beta \geqq 2$. Denoting the term on the right by $M_{m}$, we have $|\omega(t)-t| \leqq M_{m}$ for any point $t$ of an interval of order not exceeding $m$, and

$$
M_{m} \leqq \frac{1}{\beta}-\frac{1}{\alpha}+\frac{\beta-1}{\beta^{2}}-\frac{\alpha-1}{\alpha^{2}}+\frac{\beta-1}{\beta^{3}}-\frac{\alpha-1}{\alpha^{3}}+\cdots=\frac{2}{\beta}-\frac{2}{\alpha}
$$

for any $m \geqq 1$, which completes the proof.

LEMMA $4^{\prime}$.

$$
|G(y ; \alpha, \beta)-y| \leqq \frac{2}{\beta}-\frac{2}{\alpha} \quad(0 \leqq y \leqq 1) .
$$

For if $G(y)=t$, then $y=\omega(t)$, and so $|G(y)-y|=|t-\omega(t)| \leqq 2 / \beta-2 / \alpha$. This is the result required.

2.2. Definition of the nearly analytic function $\gamma(z ; \alpha, \beta)$. Set

$$
\begin{aligned}
& \Omega(t)=\omega(t) \quad(0 \leqq t \leqq 1) ; \quad \Omega(t+1)-\Omega(t)=1 \\
&(-\infty<t<\infty) . \\
& \gamma(z ; \alpha, \beta)=\gamma(z)=\omega(r) \exp \left\{2 i \pi \Omega\left(\frac{\theta}{2 \pi}\right)\right\} \\
&(0 \leqq r<\infty,-\infty<\theta<\infty)
\end{aligned}
$$

where $z=r e^{i \theta}$. Plainly $\Omega(t)$ is continuous in $(-\infty, \infty)$ as $\omega(0)=0, \omega(1)=1$, and $\gamma(z)$ is defined and one-valued for $0 \leqq|z|<\infty$ since $\gamma\left(r e^{i \theta}\right)=\gamma\left(r e^{i(\theta+2 \pi)}\right)$. From (1.16c) and (C) we deduce that $|\gamma(z)|=\omega(|z|)$,

$$
\begin{gathered}
\gamma(\alpha z)=\beta \gamma(z) . \quad \frac{\gamma\left(z^{\alpha}\right)}{\left|\gamma\left(z^{\alpha}\right)\right|}=\left\{\frac{\gamma(z)}{|\gamma(z)|}\right\}^{\beta} \quad \text { for } 0 \leqq \theta \leqq \frac{2 \pi}{\alpha} . \\
|\gamma(z+\zeta)| \leqq|\gamma(z)|+|\gamma(\zeta)| \quad(0 \leqq|z|<\infty, 0 \leqq|\zeta|<\infty),
\end{gathered}
$$

observing that $|\gamma(z+\zeta)|=\omega(|z+\zeta|) \leqq \omega(|z|)+\omega(|\zeta|)=|\gamma(z)|+|\gamma(\zeta)|$ by (B). It is easily shown that there is equality at an infinity of points $z, \zeta$. The function $\gamma(z)$ can be generalized by taking $\omega(t)=\omega\left(t ; \alpha_{1}, \beta_{1}\right), \Omega(t)=\Omega\left(t ; \alpha_{2}, \beta_{2}\right)$ where $\alpha_{1}$ and $\alpha_{2}$ or $\beta_{1}$ and $\beta_{2}$, respectively, need not be equal.

2.3. Statements of results on $\gamma(z ; \alpha, \beta)$.

LEMMA 5. The function $\gamma(z)$ has the following properties:

$$
|\gamma(z ; \alpha, \beta)-z| \leqq(2+4 \pi)\left(\frac{1}{\beta}-\frac{1}{\alpha}\right) . \quad(0 \leqq z \leqq 1) .
$$

(II) Its variation over any radius of the unit-circle is not greater than unity, over any circle $|z|=R(0<R \leqq 1)$ not greater than $2 \pi$.

(III) It satisfies the Lipschitz condition 


$$
\left|\gamma\left(z_{1}\right)-\gamma\left(z_{2}\right)\right| \leqq C_{0}\left|z_{1}-z_{2}\right|^{\lambda} \quad\left(\left|z_{1}\right| \leqq 1,\left|z_{2}\right| \leqq 1\right)
$$

where $C_{0}=1+\pi 2^{2-3 \lambda}, \lambda=\log \beta / \log \alpha$. It is, therefore, continuous.

(IV) There is an infinity of sectors $S_{j, l}(j=1,2, \cdots ; l=1,2, \cdots)$ of rings in $|z|<1$, of total area $\sum\left|S_{j, l}\right|=\pi$, such that $\gamma(z)$ is constant on any $S_{j, l}$.

(IV') The derivative $\gamma^{\prime}(z)$ exists and vanishes for almost all $z(|z| \leqq 1)$.

(V) When $\alpha<\beta^{2}$, then, given $\delta>0$, there is a non-enumerable set of circles $|z|=R(0<R<\delta)$, any of them having a non-enumerable set of quasi-poles $\zeta$ on its circumference, that is, points $\zeta$ such that $\{\gamma(z)-\gamma(\zeta)\}(z-\zeta)^{-1} \rightarrow \infty$ as $z \rightarrow \zeta$, no matter along which path $z$ approaches $\zeta$.

2.4. Proof of (I), (II), and (III). To deduce (I), we take $0 \leqq \theta<2 \pi$. We have

$$
\begin{aligned}
|\gamma(z)-z| & \leqq \omega(r)\left|e^{2 i \pi \omega(\theta / 2 \pi)}-e^{2 i \pi \theta / 2 \pi}\right|+|\omega(r)-r| \\
& \leqq 2 \pi\left|\omega\left(\frac{\theta}{2 \pi}\right)-\frac{\theta}{2 \pi}\right|+|\omega(r)-r| \leqq(4 \pi+2)\left(\frac{1}{\beta}-\frac{1}{\alpha}\right),
\end{aligned}
$$

using Lemma 4 and the inequality

$$
\left|e^{i t}-e^{i \tau}\right| \leqq|t-\tau| \quad(t, \tau \text { real }) .
$$

Now we prove (II). Fixing $\theta$, we have, with respect to the variable $r$,

$$
V_{0,1} \gamma=V_{0,1} \omega=\omega(1)-\omega(0)=1 .
$$

Fixing $r$ and taking $0=\theta_{0}<\theta_{1}<\theta_{2}<\cdots<\theta_{n}=2 \pi$, we have

$$
\begin{aligned}
V_{0,2 \pi} \gamma & \leqq 2 \pi \omega(r)\left\{\text { u.b. } \sum_{j=0}^{n-1}\left|\omega\left(\frac{\theta_{j+1}}{2 \pi}\right)-\omega\left(\frac{\theta_{j}}{2 \pi}\right)\right|\right\} \\
& =2 \pi \omega(r)\{\omega(1)-\omega(0)\} \leqq 2 \pi .
\end{aligned}
$$

To prove (III), we take $z_{j}=r_{j} e^{i \theta_{i}}, 0 \leqq \theta_{j}<2 \pi, j=1,2 ; z_{3}=r_{2} e^{i \theta}$; and $0<r_{2} \leqq r_{1}$. By (D) we have

$$
\left|\gamma\left(z_{1}\right)-\gamma\left(z_{3}\right)\right|=\omega\left(r_{1}\right)-\omega\left(r_{2}\right) \leqq\left(r_{1}-r_{2}\right)^{\lambda} \leqq\left|z_{1}-z_{2}\right|^{\lambda} .
$$

Again

$$
\begin{aligned}
\left|\gamma\left(z_{3}\right)-\gamma\left(z_{2}\right)\right| & =\omega\left(r_{2}\right)\left|e^{2 i \pi \omega\left(\theta_{1} / 2 \pi\right)}-e^{2 i \pi \omega\left(\theta_{2} / 2 \pi\right)}\right| \\
& \leqq 2 \pi \omega\left(r_{2}\right)\left|\omega\left(\frac{\theta_{1}}{2 \pi}\right)-\omega\left(\frac{\theta_{2}}{2 \pi}\right)\right|, \\
\left|\gamma\left(z_{3}\right)-\gamma\left(z_{2}\right)\right| & \leqq(2 \pi)^{1-\lambda} \omega\left(r_{2}\right)\left|\theta_{1}-\theta_{2}\right|{ }^{\lambda} .
\end{aligned}
$$

Suppose first that $\left|\theta_{1}-\theta_{2}\right| \leqq \pi$; then we have $\sin \left|\left(\theta_{1}-\theta_{2}\right) / 2\right| \geqq\left|\left(\theta_{1}-\theta_{2}\right)\right| / \pi$,

$$
\left|\theta_{1}-\theta_{2}\right| \leqq\left(\pi / 2 r_{2}\right)\left|z_{3}-z_{2}\right| \quad\left(\left|\theta_{1}-\theta_{2}\right| \leqq \pi\right),
$$


and as, by $(\mathrm{Ci}), \omega(r) \leqq r^{\lambda}$

$$
\left|\gamma\left(z_{3}\right)-\gamma\left(z_{2}\right)\right| \leqq 2^{1-2 \lambda} \pi\left|z_{3}-z_{2}\right|^{\lambda} \leqq 2^{1-2 \lambda} \pi\left|z_{1}-z_{2}\right|^{\lambda},
$$

observing that $r_{2} \leqq r_{1},\left|z_{2}\right|=\left|z_{3}\right|$, arg $z_{3}=\arg z_{1}$ and, therefore,

$$
\left|z_{3}-z_{2}\right| \leqq\left|z_{1}-z_{2}\right| \text {. }
$$

Suppose now that $\pi<\theta_{2}-\theta_{1}<2 \pi$, and take $\chi_{1}=\theta_{1}+2 \pi$; then

$$
\begin{aligned}
& 0<\chi_{1}-\theta_{2}<\pi, \quad 1 \leqq \frac{\chi_{1}}{2 \pi}<2, z_{3}=r_{2} e^{i x_{1},} \quad \Omega\left(\frac{\chi_{1}}{2 \pi}\right)=\omega\left(\frac{\theta_{1}}{2 \pi}\right)+1 \\
& \Omega\left(\frac{\chi_{1}}{2 \pi}\right)-\Omega\left(\frac{\theta_{2}}{2 \pi}\right)=\left\{1-\omega\left(\frac{\theta_{2}}{2 \pi}\right)\right\}+\omega\left(\frac{\theta_{1}}{2 \pi}\right)=\omega\left(1-\frac{\theta_{2}}{2 \pi}\right)+\omega\left(\frac{\theta_{1}}{2 \pi}\right) \\
& \leqq\left(1-\frac{\theta_{2}}{2 \pi}\right)^{\lambda}+\left(\frac{\theta_{1}}{2 \pi}\right)^{\lambda}=\left(1-\frac{\theta_{2}}{2 \pi}\right)^{\lambda}+\left(\frac{\chi_{1}}{2 \pi}-1\right)^{\lambda} \\
& \leqq 2^{1-2 \lambda} \pi^{-\lambda}\left(\chi_{1}-\theta_{2}\right)^{\lambda}
\end{aligned}
$$

if we use (1.16e), (D) and the inequality $u^{\lambda}+v^{\lambda} \leqq 2^{1-\lambda}(u+v)^{\lambda}(0 \leqq \lambda \leqq 1, u>0$, $v>0)$. Hence, using (2.44) and (2.46), we deduce that

$$
\left|\gamma\left(z_{3}\right)-\gamma\left(z_{2}\right)\right| \leqq 2 \pi \omega\left(r_{2}\right)\left|\Omega\left(\frac{\chi_{1}}{2 \pi}\right)-\Omega\left(\frac{\theta_{2}}{2 \pi}\right)\right|
$$

$$
\leqq(2 \pi)^{1-\lambda} 2^{1-\lambda} \omega\left(r_{2}\right)\left|\frac{\pi}{2 r_{2}}\left(z_{3}-z_{2}\right)\right|^{\lambda} \leqq 2^{2-3 \lambda} \pi\left|z_{1}-z_{2}\right|^{\lambda} \text {. }
$$

The result holds for $\pi<\theta_{1}-\theta_{2}<2 \pi$. Combining (2.45) or (2.47), respectively, and (2.42) we deduce (III) for $\left|z_{j}\right|>0(j=1,2)$. The inequality holds for $z_{1}=0$, or $z_{2}=0$, since $|\gamma(z)|=\omega(r) \leqq r^{\lambda}$; which completes the proof. We remark that, by (2.23), the result holds in the whole plane.

2.5. Proof of (IV) and (IV'). We consider the intervals $\left\langle T_{j}, T_{j}^{\prime}\right\rangle\left(0<T_{j}\right.$ $\left\langle T_{j}^{\prime}<1 ; j=1,2, \cdots\right)$ on which $\omega(t)$ is constant, taken in any order. We have

$$
\sum_{j=1}^{\infty}\left(T_{j}^{\prime}-T_{j}\right)=1
$$

The function $\gamma(z)$ is constant on any ring-sector $S_{j, l}$, where

$$
T_{j} \leqq r \leqq T_{j}^{\prime}, \quad 2 \pi T_{l} \leqq \theta \leqq 2 \pi T_{l}^{\prime} \quad(j=1,2, \cdots ; l=1,2, \cdots) .
$$

The area of $S_{j, l}$ is $\left|S_{j, l}\right|=\pi\left(T_{j}^{\prime}-T_{j}\right)\left(T_{l}^{\prime}-T_{l}\right)$; hence

$$
\sum_{j=1, l=1}^{\infty, \infty}\left|S_{i, l}\right|=\pi \sum_{j=1, l=1}^{\infty, \infty}\left(T_{j}^{\prime}-T_{j}\right)\left(T_{l}^{\prime}-T_{l}\right)=\pi .
$$


Now we construct a region $P_{j, l}$ interior to $S_{j, l}$ such that, given $\epsilon>0$,

$$
\left|P_{j, l}\right| \geqq\left|S_{j, l}\right|-\epsilon 2^{-j-l} \text {. }
$$

Everywhere on $P_{j, l}$ the derivative $\gamma^{\prime}(z)$ exists and vanishes identically, and we have

$$
\sum_{j=1, l=1}^{\infty}\left|P_{j, l}\right| \geqq \sum_{j=1, l=1}^{\infty}\left|S_{j, l}\right|-\epsilon \rightarrow \pi \quad \text { as } \epsilon \rightarrow 0 .
$$

Hence (IV) and $\left(I^{\prime}\right)$ are true.

\subsection{Proof of $(\mathrm{V})$. We need}

LEMMA $6\left({ }^{13}\right)$. If the function $\sigma(t)(a \leqq t \leqq b)$, nondecreasing and not reducing to a constant, is continuous and singular, then $\sigma^{\prime}(t)=\infty$ at a non-enumerable set of points of $(a, b)$.

Given $\delta>0$, we have $\omega(\delta)>\omega(0)=0$ in virtue of (Cii). Hence there exists a non-enumerable set $E$ of points $r$ of $(0, \delta)$ such that $\omega^{\prime}(r)=\infty$. Also there is a similar set $\varepsilon$ of points $\theta$ of $(0,2 \pi)$ such that $\omega^{\prime}(\theta / 2 \pi)=\infty$. We take $r \in E(r>0), \theta \in \mathcal{E}, z=r e^{i \theta}, z_{1}=r_{1} e^{i \theta_{1}}\left(r_{1}>0\right)$ and form

$$
U\left(z_{1}\right)=\frac{\gamma\left(z_{1}\right)-\gamma(z)}{z_{1}-z}=\frac{s_{1}\left(e^{i \psi_{1}}-e^{i \psi}\right)+e^{i \psi}\left(s_{1}-s\right)}{z_{1}-z}
$$

where

$$
s=\omega(r), \quad s_{1}=\omega\left(r_{1}\right) ; \quad \psi=2 \pi \omega\left(\frac{\theta}{2 \pi}\right), \quad \psi_{1}=2 \pi \omega\left(\frac{\theta_{1}}{2 \pi}\right) .
$$

Given any large $M>0$, we can find $\epsilon>0$ such that, for $\left|z_{1}-z\right|<\epsilon$,

$$
\frac{s_{1}-s}{r_{1}-r}=D>M, \quad \frac{\psi_{1}-\psi}{\theta_{1}-\theta}=d>M .
$$

We have

$$
U\left(z_{1}\right) e^{i(\theta-\psi)}=\frac{i e^{i\left(\psi_{1}-\psi\right) / 2} d s_{1} \mu\left(\theta_{1}-\theta\right)+\left(r_{1}-r\right) D}{i e^{i\left(\theta_{1}-\theta\right) / 2} r_{1} \nu\left(\theta_{1}-\theta\right)+r_{1}-r}=\frac{X}{Y}
$$

where

$$
\mu=\sin \frac{\psi_{1}-\psi}{2} / \frac{\psi_{1}-\psi}{2}, \quad \nu=\sin \frac{\theta_{1}-\theta}{2} / \frac{\theta_{1}-\theta}{2},
$$

(13) Cf. Hille and Tamarkin, loc. cit. p. 258, footnote 2; and Gilman, loc. cit., where much more detailed results are proved. For the general case see $\mathrm{H}$. Kober, loc. cit. Cf. S. Saks, Theory of the integral, 2d. ed., Warsaw, 1937, p. 128, lines 14-12 from bottom. 


$$
\begin{aligned}
\Re(X \bar{Y})= & D\left(r_{1}-r\right)^{2}+d r_{1} s_{1} \mu \nu \cos \frac{\psi_{1}-\psi-\theta_{1}+\theta}{2}\left(\theta_{1}-\theta\right)^{2} \\
& -\frac{D}{2} r_{1} \nu^{2}\left(r_{1}-r\right)\left(\theta_{1}-\theta\right)^{2} \\
& -\frac{d}{2} s_{1} \mu^{2}\left(r_{1}-r\right)\left(\theta_{1}-\theta\right)\left(\psi_{1}-\psi\right)=U+V-W-Z .
\end{aligned}
$$

Using (2.65) and (D) we have

$$
\begin{aligned}
\left|\frac{W}{Y^{2}}\right| & =\left|\frac{r_{1} \nu^{2}\left(s_{1}-s\right)\left(\theta_{1}-\theta\right)^{2}}{2 Y^{2}}\right| \leqq\left|\frac{r_{1} \nu^{2}\left(r_{1}-r\right)^{\lambda}\left(\theta_{1}-\theta\right)^{2}}{2 r r_{1} \nu^{2}\left(\theta_{1}-\theta\right)^{2}}\right| \\
& =\frac{\left|r_{1}-r\right|^{\lambda}}{2 r} \rightarrow 0 \quad\left(z_{1} \rightarrow z\right) . \\
\left|\frac{Z}{Y^{2}}\right| & =\frac{s_{1} \mu^{2}\left|r_{1}-r\right|\left(\psi_{1}-\psi\right)^{2}}{2|Y|^{2}} \leqq \frac{2^{1-2 \lambda} \pi^{2-2 \lambda} s_{1} \mu^{2}\left|r_{1}-r\right|\left(\theta_{1}-\theta\right)^{2 \lambda}}{\left(r_{1}-r\right)^{2}+\nu^{2} r r_{1}\left(\theta_{1}-\theta\right)^{2}} \\
& \leqq 2^{-2 \lambda} \pi^{2-2 \lambda} s_{1}\left(r r_{1}\right)^{-1 / 2} \mu^{2} \nu^{-1}\left(\theta_{1}-\theta\right)^{2 \lambda-1} \rightarrow 0 \quad\left(z_{1} \rightarrow z\right),
\end{aligned}
$$

as $2 \lambda=\log \beta^{2} / \log \alpha>1$. Finally we deduce that

$$
\begin{aligned}
\frac{U+V}{|Y|^{2}} & \geqq \frac{M\left\{\left(r_{1}-r\right)^{2}+r_{1} s_{1} \mu \nu\left(\theta_{1}-\theta\right)^{2} \cos \right\}}{\left(r_{1}-r\right)^{2}+r r_{1} \nu^{2}\left(\theta_{1}-\theta\right)^{2}} \\
& \geqq M \min \left\{1, \frac{s_{1} \mu \cos }{r \nu}\right\} \rightarrow \infty
\end{aligned}
$$

For $\left(u^{2}+a v^{2}\right)\left(u^{2}+b v^{2}\right)^{-1} \geqq \min (1, a / b)(a>0, b>0, u$ and $v$ real $), M \rightarrow \infty$ as $z_{1} \rightarrow z$, while $s_{1} \rightarrow \omega(r)>0, \mu \rightarrow 1, \cos \left(\psi_{1}-\psi-\theta_{1}+\theta\right) / 2 \rightarrow 1, \nu \rightarrow 1$; therefore $\Re(X / Y) \rightarrow \infty$ as $z_{1} \rightarrow z, \Re\left\{U\left(z_{1}\right) e^{i(\theta-\psi)}\right\} \rightarrow \infty$,

$$
\left|\frac{\gamma\left(z_{1}\right)-\gamma(z)}{z_{1}-z}\right| \rightarrow \infty
$$

Thus (V) is proved. A similar result holds for $\gamma(z)$ in any sector $A \leqq \theta \leqq B$ of the ring $a \leqq r \leqq b(0<a<b \leqq 1)$ if $\omega(B /(2 \pi))>\omega(A /(2 \pi))$ and $\omega(b)>\omega(a)$.

2.7. The main results on the class $\Lambda$. Suppose that $f(z)$ belongs to $\Lambda$. Write

$$
C_{f}=\underset{|z|<1}{\text { u.b. }}\left|f^{\prime}(z)\right| \text {. }
$$

The function $f(z)$ exists on the circle $|z|=1$ and is continuous for $|z| \leqq 1$. Let

$$
\eta(z ; \alpha, \beta)=f\{\gamma(z ; \alpha, \beta)\} \text {. }
$$


When $f(z)$ does not reduce to a constant then $\eta(z)=\eta(z ; \alpha, \beta)$ is a nearly analytic function, that is, it has the properties (II)-(V), with the following alterations (cf. §2.3):

The variations, referred to in (II), are not greater than $C_{f}$ or $2 \pi C_{f}$, respectively.

The constant $1+\pi 2^{2-3 \lambda}$, occurring in (III), is to be replaced by $C_{f}\left(1+\pi 2^{2-3 \lambda}\right)$. This is shown with the aid of the inequality

$$
\left|f\left(w_{2}\right)-f\left(w_{1}\right)\right|=\left|\int_{w_{1}}^{w_{2}} f^{\prime}(z) d z\right| \leqq C_{f}\left|w_{2}-w_{1}\right| .
$$

We now prove the following theorem.

Theorem 2. When both $g(z)$ and $h(z)$ belong to $\Lambda$ and $g(z)-h(z)$ does not reduce to a constant then there is a sequence of functions $H_{n}(z)(n=1,2, \cdots)$ such that, uniformly for $|z| \leqq 1, H_{n}(z) \rightarrow g(z)$ as $n \rightarrow \infty$, while $H_{n}^{\prime}(z)=h(z)$ for all $n$ and for almost all $z$. The functions $H_{n}(z)$ possess the properties (II), (III), and $(\mathrm{V})$, but the constants are $C_{g}+2 C_{h}$ and $2 \pi\left(C_{g}+2 C_{h}\right)$ in (II), $\left(C_{g}+2 C_{h}\right)$ $\cdot\left(1+2 \pi 2^{2-3 \lambda}\right)$ in (III). They have the properties (IV) and $\left(\mathrm{IV}^{\prime}\right)$ if, and only if, $h(z)$ vanishes identically.

Proof. Set $f(z)=g(z)-h(z)$, let $\beta$ be a fixed integer $(\beta \geqq 2), \alpha=\beta+n^{-1}$,

$$
H_{n}(z)=f\{\gamma(z ; \alpha, \beta)\}+h(z) .
$$

Using (2.71) and (I), we have

$$
\begin{aligned}
\left|H_{n}(z)-g(z)\right| & =|f\{\gamma(z)\}-f(z)| \leqq C_{f}|\gamma(z)-z| \\
& \leqq C_{f}(2+4 \pi)\left(\frac{1}{\beta}-\frac{1}{\alpha}\right)=\frac{C_{f}(2+4 \pi)}{\beta(n \beta+1)}
\end{aligned}
$$

which tends to zero as $n \rightarrow \infty$, uniformly for $|z| \leqq 1$. On the other hand, we have

$$
H_{n}^{\prime}(z)=\frac{d f\{\gamma(z)\}}{d z}+h^{\prime}(z)=f^{\prime}\{\gamma(z)\} \gamma^{\prime}(z)+h^{\prime}(z)=h^{\prime}(z)
$$

for almost all $z$ in $|z|<1$ since, by $\left(\operatorname{IV}^{\prime}\right), \gamma^{\prime}(z)=0$ almost everywhere. We notice that the condition $\alpha<\beta^{2}$ (see V) is satisfied. Thus we have proved the theorem.

The nearly analytic functions appear to be adapted to illustrate the Besicovitch-Saks-Zygmund Theorem (cf. Saks, loc. cit., p. 197).

2.8. On continuous functions of a real variable. A continuous function $y=F(t)$, of bounded variation over $\langle 0, a\rangle$ and such that the sum of the lengths of its intervals of invariance is $a$, is said to be basic; for instance $\omega(t)$. When $F(t)(0 \leqq t \leqq a)$ is continuous, then it can be approximated uniformly by 
basic functions of bounded variation $\left({ }^{14}\right)$. This follows at once from Theorem 2 by means of the Weierstrass approximation theorem; it is analogous to the uniform approximation by step-functions, which are singular functions as well as the basic functions of bounded variation. We can obtain similar results in some other way, and we state them without proof.

When $y=f(x)(0 \leqq x \leqq a)$ is continuous then the sum of the lengths of the intervals on which the continuous function

$$
g_{\alpha, \beta}(x)=f\left\{a \omega\left(\frac{x}{a} ; \alpha, \beta\right)\right\} \quad(0 \leqq x \leqq a)
$$

is constant equals $a$, and $g_{\alpha, \beta}(x)=0$ for almost all $x$. Yet $g_{\alpha, \beta}(x)$ is a basic function of bounded variation if, and only if, $f(x)$ is of bounded variation; otherwise, $g_{\alpha, \beta}(x)$ is not of bounded variation $\left({ }^{15}\right)$. In any case, we have

$$
g_{\alpha, \beta}(x) \rightarrow f(x) \quad \text { as } \alpha \rightarrow \beta,
$$

uniformly for $0 \leqq x \leqq a$. Similarly, uniformly for $0 \leqq x \leqq a$,

$$
f\left\{a G\left(\frac{x}{a} ; \alpha, \beta\right)\right\} \rightarrow f(x) \quad \text { as } \alpha \rightarrow \beta .
$$

The latter two results follow from the Lemmas 4 and $4^{\prime}$ without difficulty.

Birmingham, ENGLAND.

(14) Also there exists a result corresponding to Theorem 2.

(15) Compare this with Vitali's example, loc. cit., $\$ 25$. 